\title{
Chemische Wärmespeicherpumpe
}

Solartechnologie ist für die Region um den 50. Breitengrad von besonderer Bedeutung. Entscheidende Voraussetzung für ihre ganzjährige praktische Nutzung ist die Lösung des Problems der Energiespeicherung zum Ausgleich der Schwankungen der Strahlungsleistung. Eine Alternative zu den bisher gebräuchlichen sog. Latentspeichersystemen (Ausnutzung der spezifischen Wärme bzw. Schmelz-/Erstarrungswärme von Feststoffen und Flüssigkeiten) ist die bereits 1961 vorgeschlagene Ausnutzung chemischer Reaktionen und ihrer Reaktionswärmen [1]. Dabei wird eine chemische Verbindung unter Wärmeaufnahme in ihre Komponenten zerlegt, diese werden getrennt gelagert und im Bedarfsfall wieder zur (exothermen) Reaktion gebracht. Trotz der offensichtlichen Vorteile dieser Verfahrensweise (keine Wärmeisolationsprobleme, geringer Raumbedarf, Erreichbarkeit höherer Temperaturen bei Rekombination als zur Zersetzung erforderlich) sind erst in neuerer Zeit entsprechende chemische Systeme untersucht, die allerdings durchweg Zersetzungstemperaturen oberhalb $100{ }^{\circ} \mathrm{C}$ erfordern (s. hierzu Übersicht in [2]). In nördlichen Regionen sind derartige Temperaturen jedoch nur in wenigen Sommermonaten mit speziellen Kollektorsystemen erreichbar.

Um der notwendigen Bedingung niedriger Zersetzungstemperaturen $\left(<60^{\circ} \mathrm{C}\right)$ gerecht $\mathrm{zu}$ werden und so Solarenergiespeicherung mit chemischen Reaktionen auch in unseren Breiten ganzjährig zu ermöglichen, wurde ein Vakuumsystem konzipiert, in dem eine feste Hydratphase eines Salzes bei gegebener Temperatur und resultierendem Dissoziationsdruck mit der reinen $\mathrm{H}_{2} \mathrm{O}$-Gasphase im Gleichgewicht steht. Geeignete Voraussetzungen hierfür bietet z.B. das System $\mathrm{CaCl}_{2}-\mathrm{H}_{2} \mathrm{O}$ mit vier reversibel ineinander überführbaren intermediären Hydratstufen. Das vorgeschlagene Gesamtkonzept sieht vor, daß je nach den äußeren Temperaturbedingungen die reversible Reaktionsführung durch gesteuerten Ablauf der Reaktionsschritte auf drei prinzipiell verschiedenen Wegen erfolgen kann, wobei das System als, chemische
Wärmespeicherpumpe“" (A), ;,chemischer Solarenergiespeicher" (B) oder ,,chemische Wärmepumpe“ (C) arbeitet: Zwei evakuierbare Reaktionsbehälter (I, II) sind untereinander über eine Pumpenleitung mit Abzweigung zu einem Speicherbehälter verbunden. In Ausgangsstellung befindet sich in I z.B. $\mathrm{CaCl}_{2} \cdot 6 \mathrm{H}_{2} \mathrm{O}$, in II z.B. $\mathrm{CaCl}_{2} \cdot \mathrm{H}_{2} \mathrm{O}$, jeweils bei Umgebungstemperatur und zugehörigem Dissoziationsdruck. Bei Druckerniedrigung in I erfolgt isotherm Dehydratisierung, Die erforderliche Energie kann der Umgebung entnommen (A) oder durch einen Solarkollektor zugeführt (B) werden. Die $\mathrm{H}_{2} \mathrm{O}$ Gasphase wird auf den zugehörigen Wasserdampfpartialdruck komprimiert; es erfolgt Kondensation im Speichergefäß. Im Fall (C) wird die $\mathrm{H}_{2} \mathrm{O}$-Gasphase unter Druckerhöhung direkt mit dem Monohydrat in II zur Reaktion gebracht.

Im Vakuum ist die Dehydratisierung der Calciumchlorid-Hydrate eine Festkörper/ Gas-Reaktion. Die große Reaktionswärme der Gesamtreaktion (Hexahydrat $\rightleftarrows$ Monohydrat: $277,1 \mathrm{~kJ} / \mathrm{mol}$ ) resultiert aus der großen Entropieänderung. Dabei ist von besonderer Bedeutung, daß der Reaktionsablauf durch Variation des Drucks beeinflußt werden kann: Die Zersetzung einer Hydratstufe kann durch ,Abpumpen“ der Gasphase isotherm erfolgen. Da das log $\mathrm{p}-(1 / \mathrm{T})$-Diagramm für die einzelnen $\mathrm{Hy}$ dratstufen Geraden ähnlicher Steigung aufzeigt, ist die dazu erforderliche isotherme Kompressionsarbeit näherungsweise unabhängig von der Ausgangs- bzw. Umgebungstemperatur, wird aber in ihrem Betrag von einer Temperaturdifferenz zwischen den Gleichgewichtsphasen beeinflußt. Bei Temperaturerhöhung um z.B. $30{ }^{\circ} \mathrm{C}$ durch Rekombination ist die freiwerdende Reaktionswärme je nach Verfahrensart um den Faktor 10 bis $10^{2}$ größer als die erforderliche Kompressionsarbeit. Bei diesen Bedingungen ergeben sich für die Gesamtreaktion unter Einbeziehung der vier Hydratstufen für die drei möglichen Verfahrensarten die folgenden Faktoren: A: 9,6, B: 76,1 (Kollektortemperatur $30^{\circ} \mathrm{C}$ über Außentemperatur), $\mathrm{C}: 11,5$.
Das System $\mathrm{CaCl}_{2}-\mathrm{H}_{2} \mathrm{O}$ ist ein Beispiel. Für die praktische Anwendung scheint es nicht zuletzt deshalb geeignet, weil Calciumchlorid ein billiges Abfallprodukt vieler Produktionsprozesse der chemischen Industrie ist. Es ist zu erwarten, daß technologisch günstigere Systeme und Systeme mit verbesserter Energiebilanz gefunden werden. Besonders Hydrate und Ammoniakate, die in reversibler Reaktion zugänglich sind, eignen sich für einen derartigen zyklischen Reaktionsprozeß. Kinetische Untersuchungen zeigen, daß die Reaktionsgeschwindigkeit wesentlich von Porosität und Schichtdicke des Materials abhängt. Für die Praxis ist zu folgern, daß bei der Behälterkonstruktion die Füllung des Feststoffs in dünnen Schichten vorzusehen ist, wodurch gleichzeitig Reaktionsgeschwindigkeit und Wärmeübergang erhöht werden. Die Entwicklung eines Trägermaterials, das den Verteilungsgrad des Feststoffs erhöht und die Ausbildung grobkristalliner Phasen bei der Rückreaktion verhindert, ist geplant. Das Verfahren eröffnet eine Möglichkeit, Energie - auch im sog. Niedertemperaturbereich - langfristig zu speichern. Wie Beispiel B zeigt, ist die Energiebilanz dann besonders günstig, wenn die zu speichernde Wärmeenergie cine gegenüber der Umgebung erhöhte Temperatur aufweist. Daraus ergibt sich ferner die Eignung des Verfahrens zur Verwertung von Abwärme. Die spezielle Anwendung des Verfahrens in der Solartechnologie [3] liegt darin begründet, daß die in Vakuumsystemen erforderliche niedrige Zersetzungstemperatur von Kollektorsystemen selbst in der Region um den 50. Breitengrad und bei diffuser Strahlung erreicht wird.

\section{Brodalla, D. Hollenberg und R. Kniep} Universität Düsseldorf

1. Goldstein, M.: Some Physical Chemical Aspects of Heat Storage, UN Conf. on New Sources of Energy, E35 57, Rome 1961

2. Ervin, G.: J. Solid State Chem. 22, 51 (1977)

3. DBP angem., Nr. P 2810360.8 